\title{
Choice-based cardinal utility: a tribute to Patrick Suppes
}

\section{Jean Baccelli \& Philippe Mongin}

To cite this article: Jean Baccelli \& Philippe Mongin (2016): Choice-based cardinal utility: a tribute to Patrick Suppes, Journal of Economic Methodology, DOI: 10.1080/1350178X.2016.1189112

To link to this article: http://dx.doi.org/10.1080/1350178X.2016.1189112

\section{央 Published online: 29 Jun 2016.}

Submit your article to this journal \lceil

Q View related articles $\longleftarrow$

View Crossmark data $\nearrow$ 


\title{
Choice-based cardinal utility: a tribute to Patrick Suppes
}

\author{
Jean Baccelli ${ }^{\mathrm{a}}$ and Philippe Mongin ${ }^{\mathrm{b} *}$ \\ ${ }^{a}$ Département d'économie, Université de Cergy-Pontoise, cergy-pontoise, France, ${ }^{b}$ GREGHEC, \\ CNRS \& HEC Paris, Paris, France
}

(Received 19 August 2015; accepted 6 January 2016)

\begin{abstract}
We reexamine some of the classic problems connected with the use of cardinal utility functions in decision theory, and discuss Patrick Suppes' contributions to this field in light of a reinterpretation we propose for these problems. We analytically decompose the doctrine of ordinalism, which only accepts ordinal utility functions, and distinguish between several doctrines of cardinalism, depending on what components of ordinalism they specifically reject. We identify Suppes' doctrine with the major deviation from ordinalism that conceives of utility functions as representing preference differences, while being nonetheless empirically related to choices. We highlight the originality, promises and limits of this choice-based cardinalism.
\end{abstract}

Keywords: ordinal utility; cardinal utility; preference differences; representation theorems; Suppes; ordinalism; cardinalism

JEL Classification: B210; B310; CO20; D010

\section{Introduction}

This paper investigates the connection of choice data with cardinal utility functions. By the standards of today's microeconomics, the latter cannot rightfully represent the former. From the ordinal revolution of the first half of the twentieth century onwards, and despite the turn taken later by von Neumann and Morgenstern, neoclassical economists have been reluctant to allow into cardinal utility in economic theory. This reluctance is inseparable from the view, which spread out among them concurrently, that choices are the overarching kind of data to be considered. As microeconomics textbooks put it, 'cardinal utility [functions are not] needed to describe choice behavior' (Varian, 2005, p. 58), and if they nonetheless sometimes occur, they are 'simply convenient choices for a utility representation' (Mas-Colell, Whinston, \& Green, 1995, p. 50). A pivotal claim, which is generally left implicit, underlies these familiar statements: if choices are taken to constitute the empirical basis of economics, then cardinal utility loses its principled justifications. Had today's neoclassical economists not adhered to this claim, they would have felt free to rely mainly or exclusively on choice data, and nonetheless leave more scope to cardinal utility than just what convenience reasons permit. However, by the current views, this sounds like an impossible theoretical combination.

A homage to Patrick Suppes provides a good occasion to examine the claim we have singled out. ${ }^{1}$ In the post-war years, Suppes contributed to giving the nascent discipline of decision theory its operating standards, a collective undertaking in which many other prominent scholars were involved, were they among his close collaborators, like

*Email: mongin@greg-hec.com 
Luce, or more loosely connected with him, like Marschak, Savage and other followers of von Neumann and Morgenstern. All these writers assigned to decision theory the task of proving representation theorems, i.e. theorems clarifying the equivalences holding between relational statements, typically interpreted as preference statements, and numerical statements, typically interpreted as utility statements, and all of them identified this proving task with an application of the axiomatic method as could be encountered elsewhere in logic, mathematics and science. ${ }^{2}$ In this brilliant group of contributors, Suppes can be noted for two significant features. For one thing, he was generally concerned with the problem of measuring quantities, and as such conceived of the axiomatic exercise as a way to establish specific forms of measurement. In his quest for the most precise forms, he was led to emphasize cardinal representations as a suitable objective for his theorems. For another thing, being an empiricist philosopher and scientist, he took choices to be the privileged, if not unique, source of data the axiomatic exercise should pay attention to. In effect, without saying so much, Suppes was running against the ordinalist tide of neoclassical economics. We offer this not as a historical statement, but as a reinterpretation of his work, since he was neither initially trained nor primarily interested in economics as such (see his enlightening 1979 'Self-Profile', from which economics is almost absent).

In the first part of the paper, we give more flesh to the ordinalist tenets sketched at the beginning of this introduction; once again, we propose a conceptual reconstruction, and leave for others the full history of the matter. What we mean is to set a clear negative benchmark against which fruitful connections between choice and cardinal utility can be discussed. In the second part, we single out some of Suppes' contributions to the theory of cardinal representations, pertaining, respectively, to (i) choice under uncertainty, (ii) stochastic choice and (iii) choice under certainty. The third part elaborates on the conflict between these contributions and the ordinalist tenets of the first part. We highlight Suppes' suggestions on (iii), which in our view contains valuable advances. Extracting a general take-away message from his work, we end up cautiously defending the possibility of choice-based cardinal utility representations.

\section{Ordinalism and its dissenters}

Any discussion of the ordinalist tenets should begin with the celebrated discovery made by neoclassical writers at the dawn of the twentieth century. To recover the essentials of demand theory, it proved unnecessary to endow the individual consumer with a 'numerical' or 'measurable' utility function over the set of commodity baskets. A utility function that merely indicates the consumer's preference ordering over these baskets proves sufficient to deliver what can be salvaged from the old law of demand and related propositions. Before this major discovery, neoclassicals had developed demand theory from the law of decreasing marginal utilities or other assumptions that can be stated only if the utility function makes definite numerical sense. The standard account singles out Pareto (1909) for replacing this thick apparatus by a thinner one, and his followers like Slutsky (1915), Hicks and Allen (1934) and Hicks (1939) for bringing this replacement programme to completion. In this revised form of neoclassicism, utility functions would have to be ordinal, in the sense of being unique up to any increasing transformation. Henceforth, when we write that a utility function is 'ordinal', we refer to this definition. It appears to have stabilized before than the definition of a cardinal utility function did. Even relatively late in the twentieth century, neoclassical writers often adopted different words and different ideas to capture the more-than-ordinal features of a utility function. 
They said not only 'cardinal', but also 'numerical', 'measurable' and 'quantitative', being quite vague - and no doubt disagreeing between themselves - on the kind of mathematical uniqueness they had in view for the utility function. ${ }^{3}$

As Hicks (1939, p. 18) writes,

we have now to inquire whether a full theory of consumer's demand (...) cannot be built up from the assumption of a scale of preference. In constructing such a theory it will be necessary every time to reject any concept which is at all dependent on quantitative utility, so that it cannot be derived from the indifference map alone.

Beside testifying to the vagueness of ideas concerning 'quantitative utility', this comment is instructive in the following way: while Hicks means his explication to restrict the utility concept, it also turns out to restrict the preference concept. Preference is taken here to be a mere disposition to rank the objects of interest (or to classify them as being indifferent, which we include in our notion of a ranking). As Hicks goes on, 'we start off from the indifference map alone; nothing more can be allowed'. Among other things, this implies that unlike in some earlier approaches, there will be no room in the new theory for comparisons of the type ' $i$ prefers more intensely hot chocolate to coffee, than he prefers tea to herbal tea'. Such comparisons are undefined because they do something else than ranking options. Samuelson's Foundations (1947) exemplifies the glide, which we emphasize here, from the claim that utility is exclusively ordinal to the claim that preference also is. ${ }^{4}$ The Paretians never made this claim explicitly, so it remains unclear whether they would have defended it as a semantic truth about the word 'preference', or as a technical restriction, which would be imposed for theoretical or other strategic purposes.

In whichever interpretation one takes, this slimmering of the preference concept was linked to a novel emphasis on choices as the appropriate basis for demand theory. In the Manual, Pareto himself had promoted his ordinal utility analysis by arguing not only that it was logically sufficient for demand theory, but also that it made this theory more empirical, hence - in his positivistic conception - more scientific. Allegedly, this upgrading of the theory followed because the consumers' choices were the new primitives (see, e.g. 1909, III, 36bis). To reconcile Pareto's claim on choices with the one made later by Hicks, to the effect that preferences are the primitives, one must assume that the psychological data of preferences can be expressed sufficiently well by the external facts of choice. One way or another, this further reductive assumption underlay all the Paretians' work even before Samuelson, starting with his 1938 paper, gave it the famous twist of 'revealed preference'. Without elaborating here on individual positions, we wish to emphasize that all Paretians supposed a close affinity between preferences and choices, and this could only reinforce their slim conception of preferences. Indeed, to choose among options is to select some and leave others aside; from barely observing this activity, it seems easy to infer a preference understood as a ranking of options, and impossible to infer anything like a preference intensity.

Our detour by demand theory can be excused by the historical fact that it defined a benchmark for the analysis of economic decision-making in general. This benchmark is what we are interested in here. We call it ordinalism and summarize it by three reductive tenets: (1) utility functions are merely a formal representation of preferences, (2) preferences are merely a disposition to order the possible options, (3) choices merely inform the observer on how preferences order options. We take (2) as denying that a subject compares preference differences in the same structured way as he does preference levels - in other words, as denying that the relation 'I prefer more $w$ to $x$ than I 
do $y$ to $z$ ' defines an ordering. If (2) holds, (3) automatically does, but if (2) does not hold, (3) may or may not hold. That is, if preferences have more than the ordering-ofoptions property, it may or may not be the case that choices inform the observer on this extra content. The possibility of either accepting or rejecting (3) when one rejects (2) is essential to our reconstruction.

It follows from (1) and (2) together that utility functions can only be ordinal. A more traditional account of ordinalist ideas would have extracted this last claim and commented on it as follows: utility functions can only be ordinal because they merely represent preferences. We object to this account on the ground that it takes for granted the ordinalist conception of preferences as being mere rankings, and thus gives the false impression that the ordinal property of utility functions directly follows from their representational property. This virtually reduces ordinalism to claim (1) as if there were nothing more to ordinalism. By making the conception of preferences the object of a separate commitment, we make it clear that two separate claims underlie the uniqueness property of utility functions. Thus, our framework allows for the logical possibility that a utility function be merely representational and nonetheless represent preference differences, satisfying (1) but violating (2), and this will indeed be the conception we attribute to Suppes. A different objection to the more standard accounts, we think that ordinalism should be discussed not only in terms of the preference but also in terms of the choice concept, although one must be careful here not to confuse ordinalism with the revealed preference methodology (the latter being only a particular development of the former).

The present account can be compared with Mongin and d'Aspremont's briefer comments (Mongin \& d'Aspremont, 1998, pp. 385-386), which emphasize (2) as a separate claim, and the addition made by Bruni and Guala (2001, p. 24). The last authors rightly emphasize that ordinalists limited the role of psychological assumptions in economics. This feature actually derives from the present list as follows: claim (1) excludes that utility has a psychological basis in the psychological feeling of subjective satisfaction (as in most classical and early neoclassical views), claim (2) excludes part of the psychological content one may associate with the concept of preference and claim (3) minimizes the psychological content of choice information. ${ }^{5}$

To further illustrate the logical content of the three tenets, and also to prepare the upcoming comparison with Suppes, we consider a historical departure from (2) that predated his work and to some extent influenced it. In an allusive passage of the Manual (1909, IV, §32), Pareto had claimed that, when faced with four commodity baskets $w, x, y, z$, the consumer can know whether he is more satisfied passing from $x$ to $w$, or passing from $z$ to $y$. This is a striking early occurrence of the claim that comparisons can be made not only between levels of satisfaction but also between differences in these levels. However, as could be expected from the founder of ordinalism, Pareto had considered such comparisons only to exclude them; for him, they lacked sufficient 'precision' to be subjected to a scientific inquiry. But Lange (1934) spotted the curious passage and tried to develop it in a positive direction. He claimed that a subject comparing Paretian 'transitions' could be endowed with a 'cardinal' utility function. Lange's sketch of proof was unsound, as others - prominently the mathematician Alt (1936-1971) - were soon to demonstrate. But he had launched a line of research that implicitly rejected the ordinalist claim (2). Lange's analysis unfolded at the level of utility representations alone, and thus remained equivocal, but Alt made a step towards the rejection of (2) by introducing a quaternary ordering on the options, i.e. an ordering that compares pairs of options $(w, x)$ and $(y, z)$. Putting axioms on this new 
primitive term, he obtained a utility function on the options with a relevant uniqueness property, thus proving one of the first representation theorems ever, and more specifically opening the way to Suppes' work along the same line. To the extent that Alt's ordering can be interpreted as a preference ordering, he can be said to have opposed (2) and thus shaken ordinalism at the peak of its theoretical success.

Without mentioning Pareto's passage, and probably without being aware of it, Frisch $(1926,1932)$ had already formalized comparisons of 'displacements' in the commodity space by a quaternary relation. However, this is a formalization without a proper axiomatization, and in point of fact, only a brief detour in two pieces that are primarily concerned with the empirical measurement of marginal utility. We mention Frisch nonetheless because Suppes refers to Frisch, though not to Alt, whose more significant contribution became recognized only belatedly. ${ }^{6}$

The programme initiated by Lange leaves two ordinalist tenets in place. Consistently with (1), it takes utility to have no meaning per se, but only to serve as a representation device, and consistently with (3), it limits choices to providing basic ranking information. This last point calls for more detail. Lange and followers tended to take introspection to be the single source of the quaternary comparisons, and we interpret this restrictive position as being dictated by continuing adherence to (3). A comment that Allen published on Lange's work makes this connection of ideas entirely explicit:

It has been suggested that a second basic assumption can be added to the one already made [on the existence of a preference ranking of alternatives]. This assumption refers to changes in the 'intensity' of the preferences expressed by the individual. It implies, in short, that the individual can distinguish increments of preference and that he can order these increments in the same way as the preferences themselves. Here we are dealing with something quite new. The assumption cannot be expressed in terms of the individual's acts of choice; it can only be supported by introspection into one's own experience or by questioning others about their experiences. (Allen, 1935, p. 155, our emphasis)

Notice that Allen's lines also testify to the fact that Lange's readers thought of quaternary comparisons as being preference comparisons. Once a classic, Allen's (1956, pp. 669-676) text on mathematical economics discusses 'ordinal' versus 'measurable' utility along the same lines as his briefer comment on Lange.

A different argument on ordinalism became available when von Neumann and Morgenstern (1947, Appendix), and more clearly their followers, connected the timehonoured expected utility (EU) formula with a preference ordering over lotteries. This collective work, which Suppes witnessed when it was still in progress, converged to the classic theorem stating that, given relevant axiomatic conditions, the ordering has a representation in terms of this EU formula. The uniqueness clause of the theorem seemed to endow the utility function in this formula with a numerical ('measurable') property, and this raised puzzlement among post-war economists, who, by then, had been heavily exposed to ordinalist ideas. An intricate and profuse discussion resulted among them. Some claimed that von Neumann and Morgenstern (VNM) had rehabilitated the 'cardinal utility' of the classical and early neoclassicals, others, that this was not the case but they had nonetheless obtained a 'cardinal utility' function of their own, and still others, that neither was the case and that their EU representation was in fact ordinal. Although this would contextualize Suppes' work more fully, it goes beyond the scope of this paper to review the post-war debate on 'the cardinal utility which is ordinal', to use Baumol's (1958) striking words, and we refer the reader to the existing historical work in Fishburn (1989) and Moscati (2013a, 2016a). But we will briefly indicate the two key technical factors of this debate, which, respectively, 
concern the uniqueness property of the VNM utility function and its ability to induce a measurement of preference differences.

Let us then take a closer look at the VNM representation theorem. Its conclusion actually decomposes into an existence and a uniqueness part. According to the former, there exists a utility function $u$ on the final outcomes $X$ such that the preference ordering of two lotteries $P$ and $Q$ coincides with the comparison made between the expected utility values $E_{P} u$ and $E_{Q} u$ of these two lotteries. Formally, if one denotes by $\mathrm{R}$ the primitive weak preference ordering:

\section{$P \mathrm{R} Q$ if and only if $E_{P} u \geq E_{Q} u$.}

According to the latter, uniqueness part, the function $u$ is unique up to a positive affine transformation (PAT) $)^{7}$ in this representation. Formally, if $u^{\prime}$ is another utility function on $X$, the following equivalence holds:

\section{$P \mathrm{R} Q$ if and only if $E_{P} u^{\prime} \geq E_{Q} u^{\prime}$}

if and only if $u^{\prime}$ is a PAT of $u$.

A simple argument shows that the italicized clause in this representation cannot be removed. Since the VNM axiomatization assumes no more than a preference ordering on lotteries, it follows that the $E_{P} u$ representation is in fact ordinal, i.e. unique up to any increasing transformation $\varphi$. For instance, $\left(E_{P} u\right)^{2}$ or $\exp \left(E_{P} u\right)$ are as admissible representations of the preferences over lotteries as is $E_{P} u$. This observation readily entails that there is no way of reinforcing the uniqueness conclusion for $u$. For suppose that $u$ were unique to PAT unrestrictedly; then the representation $E_{P} u$ would be unique up to PAT, and not up to any increasing transformation as was just said.

This discussion suggests introducing two notions of a cardinal utility function for the remainder of the paper. Let us say that a utility function on some set of options is absolutely cardinal if it is unique up to PAT, and that it is relatively cardinal if it is unique up to PAT for a given format of representation, such as the EU format here. We will see that this contrast applies broadly. As far as VNM theory is concerned, a good deal of the post-war debate can be explained by the fact that many protagonists missed the contrast entirely. Those who claimed that VNM had rehabilitated the 'cardinal utility' of the classical and early neoclassicals were off the mark, since they ignored the new feature of VNM utility being obtained by a representation theorem, but even those who claimed that VNM had obtained a 'cardinal utility' function of their own were not always correct, since some wrongly believed that the $u$ of the representation theorem was absolutely cardinal. And those who claimed that this $u$ was ordinal missed the important point that a relatively cardinal utility obeys a stronger uniqueness restriction than an arbitrary ordinal utility function.

The other key factor of the controversy has to do with the following argument, which was often discussed in the controversy (see a classic occurrence in Luce \& Raiffa, 1957, pp. 31-34). Once the EU representation is obtained from the existence conclusion of the VNM theorem, a utility difference formalism becomes readily available. Take two equiprobable lotteries between $w$ and $x$, and between $y$ and $z$, respectively, and suppose that the preference ordering puts the first strictly above the second. Then, 


$$
\frac{1}{2} u(w)+\frac{1}{2} u(z)>\frac{1}{2} u(y)+\frac{1}{2} u(x),
$$

which is trivially equivalent to:

$$
u(w)-u(x)>u(y)-u(z) .
$$

Many participants to the debate took the comparisons of utility differences thus obtained to represent corresponding preference differences; in the particular instance, they would have concluded that the subject prefers $w$ to $x$ more than he prefers $y$ to $z$. This understanding of VNM utility differences points towards the alternative definition of a cardinal utility function as being one that can represent not only preference levels, but also preference differences, and this alternative definition was indeed present in the debate, while being unclearly related to the formal definitions of the previous paragraph. This compounded the confusion already created by the poor understanding of the formal definitions. We refrain from saying more on VNM utility differences at the present stage, since this issue will come out again when we discuss Suppes in Section 4.

As a summary on the VNM theorem, we compare it with the three ordinalist tenets. It gives a purely representational sense to the EU formula, thus supporting (1). Whether it also supports (2) is a complex issue, depending on how one views the utility difference argument of last paragraph. If one rejects (2) on the ground that VNM utility can measure preference differences, it seems obvious to reject (3), since choices among lotteries, in the plain sense of what counts as a choice, become a source of information on preference differences. The position we will attribute to Suppes concerning EU representations (more generally than the VNM representation) will consist of this twin rejection of (2) and (3). We now move to a description (Section 3) and an assessment (Section 4) of his contributions.

\section{Suppes on utility differences}

Suppes' decision-theoretic contributions respond to a unifying concern for scientific measurement. In a sequel of papers and books that culminated with the famous series of The Foundations of Measurement (starting in 1971), coauthored with Krantz, Luce and Tversky, he investigated various forms of measurement of empirical properties, and fitted them in a unified mathematical framework that permitted defining and comparing them rigorously. When he tackled decision-theoretic issues in separate pieces of research, he always emphasized that they entered the measurement framework as particular cases, and thus illustrated its heuristic fecundity.

This approach has some noticeable consequences. First of all, unlike many positivist philosophers and scientists, Suppes does not draw a sharp line between physical and psychological properties. What matters to him is whether or not they are empirical properties, and in case they are, what kind of measurement they are amenable to. With this open mind, when he discusses decision-theoretic issues, he does not discard introspection as a possible source of information and contents himself with the claim that choices are a better source. As one of his leading papers goes, 'many areas of economic and modern statistical theory do not warrant a behavioristic analysis of utility. In these domains there seems little reason to be ashamed of direct appeals to introspection' (Suppes \& Winet, 1955, p. 261). In the same vein, Luce and Suppes 
(1965, p. 273) write that introspection has been 'unduly depreciated in some of the modern literature on choice behavior'. Suppes' position is not fully explicit, but it seems to rely on the view that introspection provides empirical data no less than choices, and if choice data are altogether preferable, this is only because they support more secure forms of measurement. ${ }^{8}$

Second, Suppes practices the method of representation theorems to clarify measurement possibilities. In decision theory, this leads him to be critical of the VNM and Savage axiom systems, which he complains assume unrealistically large sets of options and preferences comparisons. His favourite manner involves taking a finite set of options and a subclass of the logically possible comparisons, even if this economy must be achieved at the expense of the elegance and generality of the representation theorems. When he cannot impose finiteness, he tries at least to avoid topological or measure-theoretic assumptions on the set of options; typically, instead of such assumptions, he introduces 'solvability conditions' among his axioms.

Third, the representation theorems that Suppes wishes for decision theory should deliver a utility difference representation (this will automatically entail a utility-level representation as a particular case). This search for a precise form of utility function does not reflect a predetermined conception of preference and choice but rather a scientific hope: to count as an empirical science, decision theory must be able at least sometimes to reach this stage of measurement, and carefully designing the observational or experimental design could perhaps bring about this result. With these general comments in place, we now sketch Suppes' contributions, using his own terms as much as possible. We will not emphasize the uniqueness problem of utility representations in the present section. This, along with other substantial comments, will be reserved for Section 4.

As Suppes and Winet (1955, p. 259) put at the outset, they are concerned with 'reviving the notion of utility differences' and believe they can do so by proving representations theorems that apply to several choice contexts at once. The authors mentioned choice under uncertainty and choice under certainty, and with the benefit of hindsight, we can add stochastic choice, which attracted Suppes' later interest. Still following Suppes and Winet, we distinguish two steps in this revival programme. Given a domain $X$ and a quaternary relation $\succcurlyeq$ defined on $X$, the first, mathematical step is to subject the relational statements $w x \succcurlyeq y z$ to axiomatic conditions that will entail a representation in terms of utility differences, namely,

$$
(*) w x \succcurlyeq y z \text { if and only if } u(w)-u(x) \geq u(y)-u(z)
$$

for some $u$ on $X$ with a relevant uniqueness property. For this statement of the mathematical problem, Suppes and Winet are indebted to the authors discussed in last section - they cite both Frisch and Lange, although not Alt, who was not yet known. The second, this time informal step, is to check that the domain $X$ and the axiomatic conditions on $\succcurlyeq$ are appropriate, given the interpretations for $\succcurlyeq$ provided by the above contexts. At this semantic level, Suppes and Winet depart from Frisch, Lange and Alt, since these predecessors had considered only one context, i.e. choice under certainty.

A preliminary comment is in order. The paper with Winet actually sets out to axiomatize an absolute difference representation, i.e.

$$
(* *) w x \succcurlyeq y z \text { if and only if }|u(w)-u(x)| \geq|u(y)-u(z)| \text {. }
$$


It is however the algebraic difference representation $\left(^{*}\right)$ that Suppes' revival programme is concerned with. We find it slightly embarrassing that the same paper contains both the clearest statement of this programme and an unrepresentative implementation of it. We will leave aside $(* *)$ until the end of Section 4 , where we argue in effect that Suppes should have axiomatized $\left(^{*}\right)$ rather then $(* *)$.

(i) Choice under uncertainty. This choice context is only briefly mentioned in the paper with Winet, but Suppes will return to it thoroughly later. If probabilities were given, Suppes would simply repeat the argument discussed in last section, and take $w x \succcurlyeq y z$ to hold if the subject weakly prefers an equiprobable lottery on $w$ and $z$ to another equiprobable lottery on $y$ and $x$. Assuming the VNM theorem to hold, this would deliver the following equivalence:

$$
w x \succcurlyeq y z \text { if and only if } \frac{1}{2} u(w)+\frac{1}{2} u(z) \geq \frac{1}{2} u(y)+\frac{1}{2} u(x),
$$

However, Suppes argues that the risk approach makes empirical sense only if 'psychological probabilities are identical with the (...) objective' ones, a very dubious assumption in his view. ${ }^{9}$ In a deep and novel insight at this formative stage of decision theory, Suppes realizes that subjective probabilities are more fundamental than lotteries and VNM theory is in fact incomplete, since it takes probabilities as given only by mathematical convention. In today's textbook terminology, Suppes subordinates the theory of risk to that of uncertainty. This is also Savage's (1972/1954) insight and we will leave it for historians to explain what the respective influences were.

Moving now to the more appropriate uncertainity context, we denote by $w A^{*} z$ (resp. $y A^{*} x$ ) the prospect of $w$ (resp. $y$ ) obtaining if $A^{*}$ occurs and $z$ (resp. $x$ ) obtaining otherwise. According to Suppes' definition, $w x \succcurlyeq y z$ holds if the subject prefers $w A^{*} z$ to $y A^{*} x$, with the event $A^{*}$ being such that for all $x^{\prime}, y^{\prime}, x^{\prime} A^{*} y^{\prime}: y^{\prime} A^{*} x^{\prime}$. In the first sketch ever made of an axiomatization of subjective probabilities, Ramsey (1926, in 1931, p. 177) had singled out events like $A^{*}$, calling them 'ethically neutral' (a strange denomination); in words, $A^{*}$ is such that a subject faced with two consequences $x^{\prime}$ and $y^{\prime}$ is equally willing to see $x^{\prime}$ realized on $A^{*}$ and $y^{\prime}$ on its complementary, or to see $y^{\prime}$ realized on $A^{*}$ and $x^{\prime}$ on its complementary. When Ramsey's axiomatic exercise is properly completed, a subjective expected utility (SEU) representation emerges and the ethically neutral event $A^{*}$ receives probability $1 / 2$, so that the prospects $w A^{*} z$ and $y A^{*} x$ can occupy the role of the two equiprobable lotteries of VNM theory, and the equivalence above holds, though with a more satisfactory interpretation of the equal probability values. A utility difference representation as in $(*)$ follows from the equivalence. Two papers by Davidson and Suppes (1956) and Suppes (1956) implement the strategy of this paragraph, while for the first time bringing Ramsey's ideas to the stage of sharp representation theorems. ${ }^{10}$

We have stressed that Suppes' axiomatic method embodies strong empirical concerns, and this is reflected in Davidson and Suppes (1956) by two departures from Ramsey's implicitly unrestricted framework. ${ }^{11}$ The set $X$ of consequences is taken to be finite, and the axioms are so devised that once the SEU representation obtains, the consequences have equally spaced utility values and combine in the EU formula with only a finite number of subjective probability values. Moreover, the set of twoconsequence prospects $w A^{*} z$ is limited to genuinely uncertain ones, i.e. it excludes $w A^{*} w$ (the certainty of $w$ ), which is justified by another empirical concern. Davidson and Suppes worry that if sure prospects enter the comparisons, they may introduce the 
distorting influence of what they call the "utility of gambling'. ${ }^{12}$ Lastly, unlike Ramsey, Davidson and Suppes restrict their axioms to the indifference part of the preference ordering on prospects, i.e. in statements of the form $w A z \sim y A x$, as against those representing the preference ordering in general, $w A z \succcurlyeq y A x$. In view of all these restrictions, their representation theorem can only be much weaker than those of Ramsey and (blurring the distinction between risk and uncertainty) VNM, but they fully endorse this implication: 'the relative weakness of the present theory is the price to be paid for making it more behavioristic' (1956, p. 26).

In Suppes' mind, an experimental stage had to follow these axiomatic preliminaries, and it took place in a separate work coauthored with an experimental psychologist (Davidson, Suppes, \& Siegel, 1957). Suppes and his collaborators tried there to identify an event that would be 'ethically neutral' across the pool of subjects. This proved to be more challenging than expected, because the subjects appeared to express preferences over the events that a fair coin lands heads rather tails, or that a fair dice rolls to an odd number rather than an even one. The experimenters adopted a dice with either of two meaningless syllables, 'ZEJ' and 'ZOJ', engraved on each of the six sides, and thus eventually generated what appeared to them to be an 'ethically neutral' event $A^{*}$. From there, they proceeded to elicit utility differences by taking consequences to be small amounts of money (ranging over cents), both positive and negative. Whenever possible, utility functions were derived from these data. In principle, an empirical verdict on SEU theory should have ensued, but the data were in fact inconclusive, as Suppes came later to recognize. ${ }^{13}$ When revisiting these experimental efforts, Luce (1979, p. 102) will also have to conclude that, careful as they were, 'they did not lead to a clear decision as to the adequacy of the expected-utility property'.

(ii) Stochastic choice. For good historical reasons, this choice context was not mentioned in Suppes and Winet, but once stochastic choice theory took off, Suppes developed an interest in the utility difference representations this theory delivers, so we have included it into his revival programme. (See the supporting comment in Suppes \& Zinnes, 1963, p. 38.) Here $w x \succcurlyeq y z$ holds in terms of a comparison between the subject's choice probabilities. Formally,

$$
w x \succcurlyeq y z \text { if and only if } p(w, x) \geq p(y, z),
$$

where $p(w, x)$ and $p(y, z)$ are the probabilities that the subject chooses $w$ over $x$ and that he chooses $y$ over $z$, given the respective menus of options $\{w, x\},\{y, z\} \subseteq X$. Relevant axiomatizations of $\succcurlyeq$ ensure that there exists a utility function $u$ on $X$ such that

$$
p(w, x) \geq p(y, z) \text { if and only if } u(w)-u(x) \geq u(y)-u(z) .
$$

Thus, as Luce and Suppes (1965, p. 334) mention, stochastic choice theory in axiomatic form provides another road to $\left(^{*}\right)$. Since the original papers by Davidson and Marschak (1959) and Block and Marschak (1960), this theory has received at least two possible interpretations. For both Suppes and Luce, the probabilities are choice frequencies when the choice task is repeated and the source of randomness lies in the choice itself, as against its psychological determining factors (among which the preferences). The alternative interpretation locates randomness in these determining factors themselves. ${ }^{14}$

Suppes witnessed the birth of the stochastic choice literature and observed its development carefully (the review in Luce \& Suppes, 1965; Section 5, is still used as a 
reference today). He also contributed to it with two novel representation theorems (Suppes, 1961). They were part of a larger, now little-known project, the originality of which was that it did not take choice probabilities as given, but derived them in a supposedly rigorous 'behavioristic' fashion. The probabilities were the asymptotic result of a learning process, which consisted of the three stages of stimulus sampling, response conditioning and reinforcement. This work was also the occasion of sketching a 'dynamic theory of (...) the acquisition of a particular set of beliefs or values', which could override the 'static' character of standard representation theorems (1961, p. 186).

(iii) Choice under certainty. This is the other choice context that Suppes and Winet mention and it is the specific object of their axiomatic work. They illustrate it by three concrete variants, each of which represents a step towards experimentation. The first hinges on the subject's willingness to pay for exchanging options, and it is curiously reminiscent of the 'money pump argument' for the transitivity of preference that Davidson, McKinsey, and Suppes (1955, p. 146) introduced in the same year. This takes $w x \succcurlyeq y z$ to hold if the subject, when endowed with both $x$ and $z$, is willing to pay at least as much to replace $x$ by $w$ as he is to replace $z$ by $y .{ }^{15}$ A closely related variant exploits the subject's willingness to work, with $w x \succcurlyeq y z$ holding if the subject, when endowed with both $x$ and $z$, is ready to work at least as much to replace $x$ by $w$ as he is to replace $z$ by $y$. As Suppes and Winet (1955, p. 260) observe, the common idea behind these two examples is that a monotonic variation in some agreed on quantity permits measuring utility differences, thus opening another road to $(*)$. Without the word and without the historical reference, the third variant leads us back to Paretian 'transitions', i.e. $w x \succcurlyeq y z$ holds if, when endowed with both $x$ and $z$, the subject is either more willing to exchange $x$ for $w$ than $z$ for $y$, or indifferent between the two exchanges. By axiomatizing $\succcurlyeq$ appropriately, one gets the utility difference representation (*), with $u$ on $X$ satisfying a relevant uniqueness property. Because Suppes and Winet derived an absolute difference representation, proper axiomatizations of $\succcurlyeq$ conforming to the third variant needed to await Suppes' later work, e.g. in Krantz, Luce, Suppes, and Tversky (1971, p. 147; see also the restatement in Köbberling, 2006, p. 381).

Each of the suggested variants has its theoretical problems. As Luce and Suppes (1965, pp. 273-275) will recognize when revisiting them, the first two resort to a quantity that does not belong to the initial set of options. One may respond to this by redefining this set, but then the objection rebounds, since the subject's preference over the new options must be separable, and this is a substantial assumption to make. ${ }^{16}$ That is, with options now defined as $(x, m)$, where $m$ is, say, a quantity of money, the subject must rank the $m$ component always in the same way regardless of the value taken by the $x$ component. This assumption may or may not be appropriate, given the objects represented by $x$. Now, the proposed scheme needs $\succcurlyeq$ also to be continuous; otherwise the subject could not determine what money amount $m^{\prime}$ makes the option $\left(y, m^{\prime}\right)$ indifferent with $(x, m)$, given two distinct $x$ and $y$. Continuity is a significant assumption to make when the options are multidimensional.

The Paretian 'transitions' variant can eschew the separability and continuity problems above, but raises other queries. We may discard the objection that to assume that the subject initially has both $x$ and $z$ violates the principle that options should be alternatives, i.e. mutually exclusive objects, for it is enough to redefine them as being pairs $(x, z)$ and think of the comparisons as being made on such pairs. ${ }^{17}$ More importantly, it is not said whether the scheme applies unrestrictedly or only in those cases in which the subject either prefers $w$ to $x$ or prefers $y$ to $z$. In the latter interpretation, the subject 
will always compare the status quo with a change for the better, and thus be faced with a genuine choice problem, but only nonnegative utility differences can be measured in this way. In the former interpretation, the subject will also compare the status quo with changes for the worse, and this permits measuring negative utility differences, but the meaning in terms of choices is not so clear. Indeed, the subject must be forced to decide between the two evils, and only an experimental context can create the conditions for a forced exchange to take place, whereas choices in the other case could in principle be observed non-experimentally. All in all, the information carried by such a decision seems to be less secure than that carried by choices in the other case.

Suppes and Winet (1955, p. 260) illustrate their use of 'transitions' by imagining a housewife faced with a pair of appliances, say a toaster and a waxer, and then confronted with the 'choice of trading the toaster for waffle iron, or the waxer for the blender'. From the corresponding passage, we conclude that Suppes envisaged changes for the worse as well as for the better. ${ }^{18}$

Suppes' writings contains regrettably little on context (iii). The article with Luce has the following comment: 'Unfortunately, we know of no experiments that have attempted to apply either of these methods to the measurement of utility differences' (1965, p. 274). This in particular indicates that he never set himself the task of experimenting with the three concrete variants of (iii). Perhaps he found work along this line too novel and difficult, perhaps he did not value it so much as he did work on (i) and (ii), which connected better with his broader theoretical projects.

We now clarify the extent to which Suppes' contributions conflict with ordinalism and try to decide whether they bring out cogent arguments against it.

\section{Suppes and ordinalism}

Suppes claimed to be a philosopher and a social scientist, not an economist. As such, he had no theoretical stake with debasing ordinalism, but he was well aware that his approach to cardinal utility went against orthodox economic theory. For instance, Luce and Suppes (1965, p. 273) write: 'if we speak of the utility difference, or the difference in preference, between pairs of alternatives, then the classical objection of economists is that choices between alternatives do not yield behavioral evidence on these differences'. Suppes also knew the economics literature well enough to recognize dissenters like Frisch and Lange. The first task of this section is to strengthen his allusive comments into an explicit position concerning ordinalism.

Since Suppes promotes the method of representation theorems and thoroughly applies it to decision theory, all his work takes claim (1) of ordinalism for granted. Claims (2) and (3), however, are potentially contentious between him and this doctrine. The statements we have proposed for these claims analytically exclude rejecting (3) while endorsing (2), so that there are only two ways to contradict ordinalism, i.e. to reject (2) and (3) or to reject (2) alone. It is not difficult to locate Suppes' position on this logical map: he opposes both (2) and (3), thus departing from the economists' received doctrine more dramatically than Lange and followers do. The last section has listed three contexts for which he considered axiomatizing 'utility differences', and reviewing them, we find that he conceived of the primitive relation $w x \succcurlyeq y z$ in terms of choices for all three contexts, and in terms of preference differences for at least contexts (i) and (iii). Here is some textual evidence for this conclusion. Suppes performed choice experiments on (i), and although he did nothing of the kind for (iii), he made nonetheless clear a choice interpretation for $w x \succcurlyeq y z$ also for this context (notice the 
word 'choice' in the housewife passage of last section). Obviously he had a choice interpretation for (ii). He made it very clear that he also supported a preference difference interpretation concerning (i): 'In the intended interpretation, $w x \succcurlyeq y z$ if and only the difference in preference between $w$ and $x$ is not greater than the difference in preference between $y$ and $z$ ' (Davidson \& Suppes, 1956, p. 262), or concerning both (i) and (iii): 'we assume that a prior satisfactory analysis of preference (as opposed to preference difference) has already been given' (Suppes \& Winet, 1955, p. 260). What is not so clear is what he thougt of the preference difference interpretation for context (ii). ${ }^{19}$ However, the ease with which he moved from the language of choice to that of preference differences - see, e.g. the quote from Luce and Suppes in the last paragraph - suggests that he viewed the former, in many circumstances, as providing good information on the latter. This is sufficient evidence to locate him beyond the stage where Lange and followers had left the rejection of ordinalism. Although by no means hostile to introspection, Suppes clearly thought that it was possible, and it would be more secure, to document preference differences in terms of choices. ${ }^{20}$

The second, more difficult task of this section is to evaluate the position we thus attribute to Suppes. We first review the three contexts again, focusing on the primitive relations that enter the representation theorems proved for them by Suppes and related writers, and we ask two critical questions concerning these relations. Do they receive a genuine choice interpretation from these contexts? And if a relation receives a genuine choice interpretation from a context, does it also receive from it a genuine interpretation in terms of preference differences?

(i) Choice under uncertainty. For definiteness, we focus our discussion on Davidson and Suppes' (1956) axiom system. As we have seen, these authors' primitive is only an indifference relation, and it is taken to hold only for two-consequence prospects, $w A z \sim y A x$, for any event $A$. Their choice interpretation for such a restricted primitive raises two questions. First, the more common procedures to reveal an indifference relation from choices approximate it by supposing that strict preferences exist, and these are not part of the primitives. We may ignore this problem as if it were purely technical. It seems indeed possible to start more generally from $w A z \succcurlyeq y A x$ and restate Davidson and Suppes' axiom system accordingly; this would just liken it to Ramsey's initial sketch. Now comes the problem that 'ethically neutral' events are elusive to the observer: a subject might have a well-defined ordering of prospects $w A z \succcurlyeq y A x$, without ever exhibiting the desired comparison, i.e. $x^{\prime} A^{*} \sim y^{\prime} A^{*} x^{\prime}$ for some $A^{*}, x^{\prime}, y^{\prime}$. If this happens, Ramsey's hope of deriving subjective probabilities from choices among prospects collapses entirely. We may, however, take Davidson, Suppes and Siegel's word that this existence problem can be overcome in practice. In sum, with relevant qualifications, we can agree that Davidson and Suppes' axioms are concerned with genuine choices and these adduce sufficient information for utility differences to be ascertainable from them.

We now attack the remaining question of whether these utility differences represent preference differences. As Davidson and Suppes preserve the essentials of the equiprobability argument used in the VNM framework, we can at the same time take a position on their work and this famous piece of decision theory. The trivial equivalence:

$$
\frac{1}{2} u(w)+\frac{1}{2} u(z) \geq \frac{1}{2} u(y)+\frac{1}{2} u(x) \Leftrightarrow u(w)-u(x) \geq u(y)-u(z) .
$$

cannot by itself guarantee that the utility differences on the right side have any meaning, let alone the desired meaning of representing preference differences. The meaning 
criterion for a property of a utility representation lies exclusively in the interpretation given to the axioms that are used to derive this representation, but neither the VNM axioms nor the more sophisticated Davidson-Suppes axioms can be interpreted in terms of preference differences. These axioms can receive a sense in terms of risk attitudes e.g. they make these attitudes independent of the lottery or the state of the world considered - and some meaning can perhaps be found to utility differences by following this semantic line, but it is clearly distinct from the meaning of interest here. This straightforward, but powerful argument was made by Luce and Raiffa (1957, pp. 31-34) when the controversy was raging over VNM utility. ${ }^{21}$ It can be refined by adding that, if utility difference could be interpreted in terms of preference differences, on top of their natural interpretation in terms of risk attitudes, this could only result from adding axiomatic material. The supplementary axioms can be spelled out in all technical detail. ${ }^{22}$ Of course, the next question will be whether such a reinforcement is purely formal or carries a plausible semantics with it. Whichever the final answer, the fact remains that, in the absence of the supplementary axioms, only the interpretation in terms of risk attitudes can be considered.

Davidson and Suppes do not even consider this objection. They may be excused on the ground that they are primarily trying to extract probabilities, and they identify utility values only with this purpose. Utility values that relate only to risk attitudes, not to preference differences, can fit such a strategy. But it is more difficult to explain Suppes' inclusion of context (i) along with context (iii) into one and the same research programme on utility differences. And elsewhere in his work, he made some definitely unguarded claims regarding the ability of $u$ to measure preference differences. For instance, Davidson et al. (1955, p. 157) bluntly write that axiom systems like the VNM one 'suggest relative simple behavioristic procedures for empirically testing degrees of preference'.

To summarize the case for context (i), this delivers a genuine choice interpretation, but lacking a suitable defence, no interpretation in terms of preference differences.

(ii) Stochastic choice. There is no question that this context brings out choice information, since the primitive term $p(w, x)$ comes with the interpretation of the frequency of a particular choice. The controversial part is how to relate the stochastic data to preferences. What we have identified as the major difficulty with (i) is still present here: preference differences do not enter the axiom system, so that the claim that the derived $u$ value permits representing them is unwarranted. The conclusion just reached for (i) holds equally well for (ii).

One may strengthen this dismissal by exploiting an intuitive argument that Luce and Suppes (1965, pp. 334-335) have impartially pointed out. In private correspondence with Luce, Savage had claimed that one could find three options $x, y$ and $z$ with the following properties: the subject wavers between $x$ and $y$, though slightly in favour of $x$, wavers between $x$ and $z$, though slightly in favour of $z$, but does not hesitate to take $z$ when the other option is $y$. If probabilities could be equated with utility differences, a contradiction would result. Translating the choice probabilities into utility differences, one would get that $u(x)-u(y)$ is a very small positive number, $u(x)-u(z)$ is another very small positive number and $u(z)-u(y)$ is a quite large positive number. This is a contradiction since

$$
u(z)-u(y)=u(z)-u(x)+u(x)-u(y)
$$


In other words, utility differences must add up, whereas choice probabilities are not expected always to do so. After a complex discussion, Luce and Suppes acknowledge that they cannot accommodate the objection entirely (see p. 337). ${ }^{23}$

One can question the link between stochastic choice and preference differences by a more direct semantic argument. In general - barring the exceptional $0-1$ case stochastic choice data do not entail the existence of an ordinal utility function. That a strongly unique $u$ exists independently of any ordinal representation is paradoxical from the perspective of utility theory. In fact, stochastic choice theory has been developed for subjects who either do not have preference orderings, or do have ones, but make implementations errors and thus deviate from their preferences at the choice level. It would be very strange to attribute an ordering of preference differences to subjects with these characteristics. ${ }^{24}$

To summarize the case for context (ii), the choice interpretation is unproblematic, but the interpretation in terms of preference differences fails, and this time, we suggest, for deep semantic reasons, and not simply for lack of a proper justification.

(iii) Choice under certainty. We begin by a technical move, i.e. the dismissal of the absolute difference representation $(* *)$ that Suppes and Winet (1955) axiomatically derive. They need the formidable axiom (labelled A5 in their paper):

$$
x y \succcurlyeq y x \text { for all } x, y \in X .
$$

In preference terms, this would entail that the subject is indifferent between moving up or down the utility scale, which seems absurd. Some empirical measurements fit the axiom and ensuing representation very well, for instance those involving the distance concept, and it remains a mystery why Suppes did not mention them in his paper instead of connecting his axiomatic with a 'revival' programme that is ostensibly concerned with preferences. A possible explanation is that he was primarily interested in this programme, but did not yet have the right theorem for his purpose. As we mentioned, axiom systems for $(*)$ appear only in his later work. The crucial axiom in these systems is:

(A) $w x \succcurlyeq y z$ if and only if $z y \succcurlyeq x w$ for all $x, y, z, w \in X$,

which is of course much weaker than Suppes and Winet's A5.

Having thus cleared the ground, we discuss our two questions in terms of comparisons of Paretian 'transitions'. We have left pending the problem that these comparisons raise when it comes to interpreting them in terms of choices. In one variant, the observer collects comparisons only when both 'transitions' are for the better, and in the other, he collects them in all cases. Let us consider the more encompassing variant first. When the subject does not spontaneously depart from the status quo, the observer will have to tell him that he should do so, and force him if necessary. This is a forced choice all right, but is it less of a choice for that? A purist who would complain that the choice information is blurred by the intervention would have to take a sceptical stand on experimental work in decision theory as a whole, since the instructions that experimenters need to give to their subjects are similarly verbal and authoritarian. They create artificial situations that are only remotely related to the subjects' own experiences, and the answers given for these situations nonetheless count as being representative of the subjects' choices. To deny that would be to dismiss basic experiments, such as Allais' and Kahneman and Tversky's, whose significance is well established in 
decision theory. We do not see why an experiment on swapping objects, as in the housewife example, would strike subjects as being stranger than standard experiments on lotteries, which involve the possibly unfamiliar concept of a numerical probability. The consistent purist will have to go as far as to claim that only non-induced choices count, as is supposedly the case with market observations. We interpret Lange's and Allen's claims regarding introspection as reflecting this drastic stand, which neoclassical economist often endorsed both before and after these writers. Here Suppes' position as an external observer of economics appears to be a clear advantage.

Another line of argument is available anyhow, which makes it less necessary to discuss experimentation. For $(*)$ to obtain, axiom (A) must hold, and since it is needed anyhow, one may use it as well to convert the information on positive differences into information on negative differences. That is, instead of letting the subject decide between 'transitions' for the worse, one will apply (A) to the more natural comparison made for the better that results from reversing the order of the 'transitions'. Notice that (A) is powerful enough also to cover comparisons between a 'transition' for the better and a 'transition' for the worse. ${ }^{25}$ A philosophical discussion may then ensue to decide whether (A) makes sense only for a rational agent or as a matter of definition; this discussion would be reminiscent of the classic one concerning the transitivity of preference, which has been defended in both ways.

We have these two principled ways of defending a choice interpretation for the primitive relation $w x \succcurlyeq y z$ when context (iii) prevails, but we must acknowledge the complication of motivating the choices in the course of an experiment. Following the received methodology of experimental economics, if Suppes' housewife is to answer truthfully whether she chooses trading the toaster for the waffle iron, or trading the waxer for the blender, she must be materially motivated to do so. The purist of our previous discussion may change tack and argue in this more pragmatic way. However, it is unclear whether the motivation problem is worse here than it is in basic choice experiments such as Allais' or Kahneman and Tversky's, and the purist may have again to extend his critique farther than he means to.

We now move to the question of whether the relation $w x \succcurlyeq y z$ can be interpreted in terms of preference differences. This is a priori unproblematic, because the axiom systems are geared at this interpretation, unlike those proposed for contexts (i) and (ii), but an a posteriori check may be desirable. It would consist in selecting a system from Suppes' late work, or even better from a recent catalogue of such systems like the one Köbberling (2006) offers, and checking that each axiom in turn can receive a plausible semantics in terms of preference differences. We eschew this task here and just claim that it can be carried out.

To summarize the case for context (iii), it appears to pass both interpretative tests, unlike the others.

The rejection of both ordinalist tenets (2) and (3) seems to be warranted at long last, but we must now explain what kind of utility functions will come out of this rejection and the simultaneous acceptance of tenet (1). In Section 2, we have clarified the uniqueness of the VNM utility function $u$ on $X$ by saying that it is only relatively cardinal. The same restriction holds not only for the utility functions obtained by other representation theorems for context (i), such as that of Davidson and Suppes, but also for the utility functions obtained by the very different theorems proved for contexts (ii) and (iii). Since the last context has emerged as the only one of relevance, we consider it exclusively from now on. 
Like the VNM representation theorem, those proved for (iii) have an existence and a uniqueness conclusion. According to the former, there exists a utility function $u$ on $X$ such that

$$
w x \succcurlyeq y z \text { if and only if } u(w)-u(x) \geq u(y)-u(z),
$$

and according to the latter, $u$ is unique to PAT in this utility difference representation. This specific relative uniqueness conclusion actually depends on having included relevant solvability assumptions into the axioms - Suppes' way - or making sufficiently strong domain assumptions - the more standard contemporary way - but we may gloss over this technicality, and just concentrate on the fact that the format restriction is inevitable, exactly as it was in the VNM case. ${ }^{26}$

The previous equivalence can be transformed into

$$
w x \succcurlyeq y z \text { if and only if } \varphi(u(w)-u(x)) \geq \varphi(u(y)-u(z)),
$$

where $\varphi$ is any increasing transform of $u$, and this states exactly what the generic form of the representation of the quaternary relation is. We can illustrate the consequences for the uniqueness of $u$ by taking $\varphi=\exp$, so that

$$
w x \succcurlyeq y z \text { if and only if } \exp (u(w)-u(x)) \geq \exp (u(y)-u(z)) .
$$

If we define $u^{\prime}=\exp u$, this equivalence becomes

$$
w x \succcurlyeq y z \text { if and only if } \frac{u^{\prime}(w)}{u^{\prime}(x)} \geq \frac{u^{\prime}(y)}{u^{\prime}(z)},
$$

and we have checked that, by deviating from the utility difference format, one can find a utility function $u^{\prime}$ on $X$ that is not a PAT of $u$, but can nonethless serve to represent the primitive relation. In brief, even with a direct axiomatization of preference differences, the resulting utility function on $X$ can only be relatively cardinal.

Does this fact diminish our conclusion that a utility function can combine the three attributes of being purely representational, representing preference differences and having a choice basis? We do not think so. There is no analytic connection between the property of representing preference differences and of being cardinal absolutely rather than relatively, so that we can take the fact in question as making the conclusion more precise instead of contradicting it. It is true that from the point of view of measurement theory, an absolutely cardinal utility function would be a more satisfactory result. If it could be obtained by representation theorems in decision theory, some measurements in this field would enjoy the respectable status of temperature measurement. There are occasional vacillations in Suppes' and Luce's statements of the uniqueness of utility functions, and they suggest that they might have been misled by such physical analogies. $^{27}$ With the benefit of hindsight, one may doubt that decision theory will ever reach this higher measurement stage, and the hope seems also forlorn in the related field of psychophysics. For instance, Falmagne's (Falmagne, 2002) book on psychophysics directly defines a utility difference representation in terms of the generic form $\varphi(u(w)-u(x))$. 


\section{Conclusion}

On the occasion of an homage to Patrick Suppes, we have revisited some classic controversies of theoretical economics on ordinal and cardinal utility functions. No doubt a full investigation of this contrast should involve one in considering not only Suppes and his group of collaborators, but also Allais (1994) and Harsanyi (1955), who developed different brands of ideas about cardinal utility, and writers in the classical and early neoclassical tradition, who did not think of utility functions as being obtained by representation theorems. Despite these lacunas, we hope to have brought some conceptual clarity to a still poorly understood debate. By identifying three distinctive claims in ordinalism, which is not usually done, we have been able to contrast Suppes' choicebased cardinalism from Lange's introspection-based form of this doctrine, and by carefully attending to the theoretical and empirical differences between the choice contexts in which Suppes' cardinalism could possibly be implemented, we have finally been able to retain one of his suggestions.

\section{Acknowledgements}

The authors are grateful to the editors of the Journal of Economic Methodology for inviting this paper, and the second author thanks them for allowing him to pay a personal tribute to Patrick Suppes' leadership and inspiration. The paper was completed when this author was visiting Wissenschaftskolleg zu Berlin. The authors have greatly benefited from a continuous dialogue with Ivan Moscati, as well from Mikaël Cozic's insightful comments.

\section{Notes}

1. To our knowledge, the present paper and Moscati's (2016b) are the first to discuss Suppes' utility theory in any detail. Previous homages have emphasized his measurement theory and probability theory, and thus touched on our topic only indirectly (Luce, 1979; Rosenkrantz, 1979).

2. Mongin (2003) has questioned this identification of the axiomatic method with representation theorems. We will however take it for granted here.

3. See Moscati's (2013a, 2013b, 2016a) thorough account of how the cardinal versus ordinal distinction stabilized in economics and decision theory.

4. The Foundations uses 'ordinal utility' and 'ordinal preference' interchangeably, and the index of the book refers to both expressions in a single entry (labelled after the latter).

5. On a different score, we do not need to extend our notion of 'ordinalism' to the collective form prevailing in social choice theory and social ethics. The word has been used there quite extensively, still assuming (2) without saying, to deny that interpersonal comparisons of utility are possible (see, e.g. Arrow, 1973, p. 253).

6. On the distinction between a formalization and an axiomatization, see Mongin (2003). Chipman (1971, pp. 327-329) confirms Alt's precedence in axiomatizing cardinal utility (without the word 'cardinal') for the first time. Moscati (2013a) examines how ideas of comparing 'transitions' spread out among economists.

7. A positive affine transformation is of the form $f(x)=a x+b$, with $a>0$.

8. Whether or not this position conflicts with behaviourism becomes a terminological issue. In his self-retrospective, Suppes claims to adhere to a form of 'methodological behaviorism (...) wholly compatible with mentalistic concepts' (Suppes, 1979, p. 34).

9. This quote is from Davidson and Suppes (1956, p. 159). A similar passage can be found in Suppes and Winet (1955, p. 259).

10. See also Bradley's (2004) reconstruction of Ramsey in fully contemporary style.

11. By a curious contrast, Suppes (1956) defines the quaternary relation $\succcurlyeq$ on a set even larger than Ramsey's, i.e. the set of probability mixtures of prospects. As Suppes is well aware of, this contravenes to his principle of taking small domains for $\succcurlyeq$ 
12. The utility of gambling (which Suppes understands somewhat differently from VNM) is the topic of Royden, Suppes and Walsh (1959).

13. One problem was that the experimental model allowed for an 'error' in the subject's responses, and this turned out also to permit violations of the SEU axioms. Suppes and Walsh (1959) tried to circumvent the problem, but their results were not clear-cut either.

14. For surveys that emphasize this duality, see de Palma and Thisse (1987) and Fishburn (1998).

15. Rosenkrantz (1979, p. 117) interestingly connects this variant with the idea of 'dollar vote' in social choice theory.

16. Fishburn (1970, Chapter 6) restates the problem in this more sophisticated way.

17. This objection appears in Fishburn (1970, Chapter 6).

18. We conclude this from the expression 'due account being taken of the algebraic sign of the difference' (Suppes \& Winet, 1955, p. 260). This expression recurs in Luce and Suppes (1965, p. 274).

19. See Luce and Suppes' (1965, p. 334) convoluted comment on two forms of 'strength of preference' in context (ii). This is part of a passage where they present a counterexample by Savage, which we are going to discuss.

20. When commenting on Lange, Alt (1936, in 1971, p. 425) and Zeuthen (1937, p. 237) had passingly contemplated the possibility of collecting choice evidence on preference differences.

21. Allen (1956, pp. 674-675) makes the same point using numerical examples.

22. See Bouyssou and Vansnick's (1990) clear summary.

23. Savage takes $x$ to be a pony, $y$ to be an ordinary bicycle and $z$ to be a sophisticated bicycle. The subject, a boy, cannot make his mind between $x$ and $y$, or between $x$ and $z$, but does not hesitate anymore when comparing $z$ and $y$.

24. We have followed Luce's and Suppes' usual view of choice probabilities. In the alternative conception, randomness takes place prior to the choice level, and is often understood as having to do with what ordering the subject will implement in his choices. It is no more plausible in this conception that utility differences capture preference differences.

25. From (A), if $w x \succcurlyeq y y$, then $y y \succcurlyeq x w$, whence $w x \succcurlyeq x w$ by transitivity.

26. See Basu (1982) for the standard contemporary way of reaching the relative uniqueness restriction. Note that a domain restriction is also embodied in VNM theory, as it postulates a set of lotteries, which are highly structured objects.

27. A somewhat gross example appears in the following passage: 'the various systems developed to represent the expected utility hypothesis end up with the result that utility is measured on an interval scale' (Luce \& Suppes, 1965, p. 284). A utility function defined on an interval scale is absolutely cardinal. This is however a rare example; compare with the entirely correct formulation in Davidson and Suppes (1956).

\section{References}

Allais, M. (1994). Cardinal utility. In M. Allais \& O. Hagen (Eds.), Cardinalism: A fundamental approach (Chapter 2, pp. 31-64). New York, NY: Springer.

Allen, R. G. D. (1935). A note on the determinateness of the utility function. The Review of Economic Studies, 2, 155-158.

Allen, R. G. D. (1956). Mathematical economics. London: MacMillan.

Alt, F. (1936). Über die Messbarkeit des Nutzens. Zeitschrift für Nationalökonomie, 7, 161-169. Translated as "On the Measurability of Utility," in Chipman, Hurwicz, Richter, and Sonnenschein (1971), 2, ch. 20, 414-431.

Arrow, K. J. (1973). Some ordinalist-utilitarian notes on Rawls's theory of justice. The Journal of Philosophy, 70, 245-263.

Basu, K. (1982). Determinateness of the utility function: Revisiting a controversy of the thirties. The Review of Economic Studies, 49, 307-311.

Baumol, W. J. (1958). The cardinal utility which is ordinal. The Economic Journal, 68, 665-672.

Block, H. D., \& Marschak, J. (1960). Random orderings and stochastic theories of responses. In I. Olkin, S. Ghurye, W. Hoffding, W. Madow, \& H. Mann (Eds.), Contributions to probability and statistics. Essays in honor of harold hotelling (pp. 97-132). Stanford: Stanford University Press. 
Bouyssou, D., \& Vansnick, J. C. (1990). "Utilité cardinale" dans le certain et choix dans le risque ["Cardinal utility" and risky choice]. Revue économique, 41, 979-1000.

Bradley, R. (2004). Ramsey's representation theorem. Dialectica, 58, 483-497.

Bruni, L., \& Guala, F. (2001). Vilfredo pareto and the epistemological foundations of choice theory. History of Political Economy, 33, 21-49.

Chipman, J. S. (1971). Introduction to Part II. In Chipman, J. S., Hurwicz, L., Richter, M. K., \& Sonnenschein, H. F. (Eds.), Preferences, utility, and demand (Vol. 2, pp. 321-331). New York, NY: Harcourt Brace Jovanovich Inc.

Davidson, D., \& Marschak, J. (1959). Experimental tests of a stochastic decision theory. In C. Churchman, \& P. Ratoosh (Eds.), Measurement: Definitions and theories (pp. 233-269). New York, NY: Wiley.

Davidson, D., McKinsey, J., \& Suppes, P. (1955). Outlines of a formal theory of value, I. Philosophy of Science, 22, 140-160.

Davidson, D., \& Suppes, P. (1956). A finitistic axiomatization of subjective probability and utility. Econometrica, 24, 264-275.

Davidson, D., Suppes, P., \& Siegel, S. (1957). Some experiments and related theory on the measurement of utility and subjective probability. Stanford: Stanford University Press.

de Palma, A., \& Thisse, J.-F. (1987). Les modèles de choix discrets [Discrete choice models]. Annales d'économie et de statistique, 14, 151-190.

Falmagne, J. C. (2002). Elements of psychophysical theory. Oxford: Oxford University Press.

Fishburn, P. C. (1970). Utility theory for decision making. New York, NY: Wiley.

Fishburn, P. C. (1989). Retrospective on the utility theory of von Neumann and Morgenstern. Journal of Risk and Uncertainty, 2, 127-157.

Fishburn, P. C. (1998). Stochastic utility. In S. Barberà, P. Hammond, \& C. Seidl (Eds.), Handbook of utility theory (Vol. 1, Chapter 7, pp. 273-320). Dordrecht: Kluwer Academic Press.

Frisch, R. (1926). Sur un problème d'économie pure. Norsk Mathema-tish Forenings Skrifter, 1 (16), 1-40. Translated as "On a Problem in Pure Economics", in Chipman, Hurwicz, Richter, and Sonnenschein, 2, ch. 19, 386-423.

Frisch, R. (1932). New methods of measuring marginal utility. Tübingen: Mohr.

Harsanyi, J. C. (1955). Cardinal welfare, individualistic ethics, and interpersonal comparisons of utility. Journal of Political Economy, 63, 309-321.

Hicks, J. R. (1939). Value and capital. Oxford: Clarendon Press.

Hicks, J. R., \& Allen, R. G. D. (1934). A reconsideration of the theory of value. Part I. Economica, 1, 52-76.

Köbberling, V. (2006). Strength of preference and cardinal utility. Economic Theory, 27, 375-391.

Krantz, D. H., Luce, R. D., Suppes, P., \& Tversky, A. (1971). Foundations of measurement (Vol. I). New York, NY: Academic Press.

Lange, O. (1934). The determinateness of the utility function. The Review of Economic Studies, $1,218-225$.

Luce, R. D. (1979). Suppes' contributions to the theory of measurement. In Bogdan, R. J. (Ed.), Patrick Suppes (pp. 93-110). Dordrecht: Reidel.

Luce, R. D., \& Raiffa, H. (1957). Games and decisions: Introduction and critical survey. New York, NY: Wiley.

Luce, R. D., \& Suppes, P. (1965). Preference, utility, and subjective probability. In R. Luce, R. Bush, \& E. Galanter (Eds.), Handbook of mathematical psychology (Vol. III, pp. 229-441). New York, NY: Wiley.

Mas-Colell, A., Whinston, M. D., \& Green, J. R. (1995). Microeconomic theory. Oxford: Oxford University Press.

Mongin, P. (2003). L'axiomatisation et les théories économiques [The axiomatic method and economic theories]. Revue économique, 54, 99-138.

Mongin, P., \& d'Aspremont, C. (1998). Utility theory and ethics. In S. Barberà, P. Hammond, \& C. Seidl (Eds.), Handbook of utility theory (Vol. 1, Chapter 10, pp. 371-481). Dordrecht: Kluwer Academic Press.

Montesano, A., Zanni, A., Bruni, L., Chipman, J. S., \& Mclure, M. (2014). Manual of political economy. Oxford: Oxford University Press.

Moscati, I. (2013a). How cardinal utility entered economic analysis: 1909-1944. The European Journal of the History of Economic Thought, 20, 906-939. 
Moscati, I. (2013b). Were Jevons, Menger, and Walras really cardinalists? On the notion of measurement in utility theory, psychology, mathematics, and other disciplines, 1870-1910. History of Political Economy, 45, 373-414.

Moscati, I. (2016a). How economists came to accept expected utility theory: The case of Samuelson and Savage. Journal of Economic Perspectives, 30, forthcoming.

Moscati, I. (2016b). Measurement theory and utility analysis in SuppesÕ early work, 1951-1958. Journal of Economic Methodology, 23, XY-XZ.

Pareto, V. (1909). Manuel d'économie politique [Manual of political economy]. Paris: Giard \& Brière.

Ramsey, F. P. (1931). Truth and probability. In R. B. Braithwaite (Eds.), The foundations of mathematics and other logical essays (Chapter 7, pp. 156-198). London: Kegan Paul, 1931.

Rosenkrantz, R. D. (1979). Suppes on probability, utility, and decision theory. In Bogdan, R. J. (Ed.), Patrick Suppes (pp. 111-129). Dordrecht: Reidel.

Royden, H., Suppes, P., \& Walsh, K. (1959). A model for the experimental measurement of the utility of gambling. Behavioral Science, 4, 11-18.

Samuelson, P. A. (1938). A note on the pure theory of consumer's behavior. Economica, 5, 61-71.

Samuelson, P. A. (1947). Foundations of economic analysis. Cambridge, MA: Harvard University Press.

Savage, L. J. (1972/1954). The foundations of statistics. New York, NY: Dover.

Slutsky, E. (1915). Sulla teoria del bilancio del consumatore. Giornale degli economisti e rivista di statistica, 51(1), 1-26. Translated as "On the Theory of the Budget of the Consumer", in Readings in Price Theory, ed. by G. J. Stigler and K. E. Boulding, 27-56. Homewood, IL: Irving, 1952.

Suppes, P. (1956). The role of subjective probability and utility in decision-making. In J. Neyman (Eds.), Proceedings of the Third Berkeley Symposium on mathematical statistics and probability (Vol. 5, pp. 61-73). Berkeley: University of California Press.

Suppes, P. (1961). Behavioristic foundations of utility. Econometrica, 29, 186-202.

Suppes, P. (1979). Self-Profile. In R. J. Bogdan (Ed.) Patrick Suppes (pp. 3-56). Dordrecht: Reidel, 1971.

Suppes, P., \& Walsh, K. (1959). A non-linear model for the experimental measurement of utility. Behavioral Science, 4, 204-211.

Suppes, P., \& Winet, M. (1955). An axiomatization of utility based on the notion of utility differences. Management Science, 1, 259-270.

Suppes, P., \& Zinnes, J. (1963). Basic measurement theory. In R. D. Luce, R. R. Bush, \& E. Galanter (Eds.), Handbook of mathematical psychology (Vol. I, pp. 1-76). New York, NY: Wiley.

Varian, H. R. (2005). Intermediate microeconomics. New York, NY: Norton.

von Neumann, J., \& Morgenstern, O. (1947). The theory of games and economic behavior. Princeton: Princeton University Press.

Zeuthen, F. (1937). On the determinateness of the utility function. The Review of Economic Studies, 4, 236-239. 